ARTICLE

https://doi.org/10.1038/s41467-019-11829-y

\title{
Ultrabright gap-enhanced Raman tags for high-speed bioimaging
}

Yuqing Zhang ${ }^{1,2}$, Yuqing $\mathrm{Gu}^{1}$, Jing $\mathrm{He}^{1}$, Benjamin D. Thackray ${ }^{1} \&$ Jian Ye (1) 1,3,4

Surface-enhanced Raman spectroscopy (SERS) is advantageous over fluorescence for bioimaging due to ultra-narrow linewidth of the fingerprint spectrum and weak photo-bleaching effect. However, the existing SERS imaging speed lags far behind practical needs, mainly limited by Raman signals of SERS nanoprobes. In this work, we report ultrabright gapenhanced Raman tags (GERTs) with strong electromagnetic hot spots from interior subnanometer gaps and external petal-like shell structures, larger immobilization surface area, and Raman cross section of reporter molecules. These GERTs reach a Raman enhancement factor beyond $5 \times 10^{9}$ and a detection sensitivity down to a single-nanoparticle level. We use a $370 \mu \mathrm{W}$ laser to realize high-resolution cell imaging within $6 \mathrm{~s}$ and high-contrast (a signalto-background ratio of 80 ) wide-area $\left(3.2 \times 2.8 \mathrm{~cm}^{2}\right)$ sentinel lymph node imaging within $52 \mathrm{~s}$. These nanoprobes offer a potential solution to overcome the current bottleneck in the field of SERS-based bioimaging.

\footnotetext{
${ }^{1}$ State Key Laboratory of Oncogenes and Related Genes, School of Biomedical Engineering, Shanghai Jiao Tong University, Shanghai 200030, P. R. China. 
S urface-enhanced Raman scattering (SERS) technique is of crucial scientific and practical interest in many fields, such as chemistry, physics, biology, and medicine ${ }^{1-7}$. SERS tags, as one of the most popular optical labels ${ }^{8-10}$, have exhibited great potential for bioimaging with advantanges of the unique fingerprint vibrational spectrum and the ultra-narrow spectral linewidth. While fluorescent tags can be bright, they typically exhibit poor photostability, allow limited multiplexing due to their broad and overlapped fluorescence spectra, and suffer the autofluoscence from biological tissues in in vivo applications. SERS tags have shown great prospects for in vivo bioimaging applications such as bright optical guidance for intraopeative detection of cancer margins, residual cancerous foci, microscopic or disseminated tumors, and sentinel or metastasized lymph node $e^{6,11-18}$. However, the existing SERS imaging speed lags far behind clinical needs. For example, it typically takes hours to acquire a wide-area Raman in vivo image ${ }^{13,16}$. The current bottlenecks for high-speed SERS bioimaging mainly include the overall Raman signals (instead of the Raman enhancement factor) of SERS tags and imaging method of Raman system.

There have been a lot of efforts to design and synthesize SERS tags that can generate bright and stable SERS signals ${ }^{19,20}$. The intensity of SERS tags largely rely on the creation of electromagnetic hot spots, i.e., spatially localized regions with extremely strong electromagnetic fields ${ }^{21}$. In addition to the creation of sharp tips and rough surfaces through morphologically controlled synthesis, hot spots can be constructed effectively by creating nanogaps on or within metal nanostructures ${ }^{19,22-25}$. Although Raman signal amplifcation has been studied on single nanoparticles (e.g., spherical, polyhedral, and nanostar shapes) and aggregates (e.g., dimers and trimers) with varying sizes and surface morphologies 26,27 , most structures suffer from poor controllability in creating uniform hot spots and generating stable SERS signals. Recently, we reported a strategy in designing and synthesizing gap-enhanced Raman tags with a smooth external shell (S-GERTs for brevity), which are composed of metallic core-shell nanoparticles (NPs) with an internal nanogap and embedded aromatic dithiol (e.g., 1,4-benzenedithiol, 1,4-BDT) Raman reporters ${ }^{13,28-30}$. Compared to the nanogaps randomly formed in NP aggregates, S-GERTs provide strong, uniform and stable SERS signals by embedding Raman molecules in the interior nanogap hot spots ${ }^{11,12}$. More efforts have been recently spent to understand and further improve the performance of these NPs ${ }^{31-39}$. However, the overall Raman signal of these S-GERTs is still not strong enough to reach high-speed imaging. For example, the Raman imaging using S-GERTs still takes more than $40 \mathrm{~min}$ over a scanning area of $1 \mathrm{~cm} \times 1 \mathrm{~cm}^{40}$, which is not conducive to real-time rapid imaging during surgery. Therefore, we need to engineer new SERS tags for more versatile and highspeed imaging purpose.

In this work, we report a version of GERTs with petal-like shell structures (P-GERTs for brevity) for high-speed Raman imaging. By utilization of strong electromagnetic hot spots from interior sub-nanometer gaps and external petal-like shell structures, a larger surface area for molecular immobilization, and a larger Raman cross section of reporter molecules, these sub-100 nm sized P-GERTs show two orders of magnitude stronger Raman signals than S-GERTs. P-GERTs can reach a Raman enhancement factor (EF) beyond $5 \times 10^{9}$ and a detection sensitivity down to a single-NP level. With the fact that the amount of Raman reporters (4-nitrobenzenethiol, 4-NBT) on gold $(\mathrm{Au})$ cores is a key to control shell morphology and SERS performance of P-GERTs, we have suggested a potential growth mechanism of forming petal-like shell. Due to the ultra-strong Raman signal of P-GERTs, ultrafast imaging system and data processing method, high-speed and high-resolution cell imaging (2500 pixels) can be obtained within $6 \mathrm{~s}$, and high-contrast wide-area $\left(3.2 \times 2.8 \mathrm{~cm}^{2}\right)$ in vivo sentinel lymph node (SLN) imaging can be obtained within $52 \mathrm{~s}$. Additionally, a variety of P-GERTs can be obtained to realize multiplexed cell imaging by facilely changing Raman reporters in the external nanogaps. These P-GERTs offer a potential solution to overcome the current bottleneck in the field of SERS-based bioimaging.

\section{Results}

P-GERTs vs. S-GERTs. S-GERTs and P-GERTs are both synthesized in a similar wet chemistry process but exhibit quite distinct particle morphology, far- and near-field plasmonic properties, and SERS performance due to the decoration with different interior Raman reporters. It is shown from the schematic diagrams that core-shell structured S-GERTs exhibit a continuous interior nanogap and a smooth external shell surface, where both Raman reporters 1,4-BDT can be adsorbed (Fig. 1c). In contrast, P-GERTs exhibit a continuous internal nanogap but a petal-like shell structure with a rough surface, both decorated with 4-NBT reporters (Fig. 1a). 4-NBT has a similar molecular structure with 1,4-BDT except the nitro group replacing the second thiol group in 1,4-BDT. In a typical experiment of preparing P-GERTs, $22 \mathrm{~nm}$ uniform-sized Au cores were modified with 4 -NBT molecules via $\mathrm{Au}-\mathrm{S}$ bonds and were centrifuged to remove the excess amount of molecules, and then the obtained 4-NBT modified Au cores were utilized as seeds for further growth of the Au shell. TEM images confirm that P-GERTs consist of a $\mathrm{Au}$ core-rough shell structure spaced by a uniform interior gap with a typical size of $\sim 0.7 \mathrm{~nm}$ (indicated by a red arrow in Fig. 1b), which is determined by the thickness of the monolayer of embedded 4 -NBT molecules ${ }^{15,29}$. Unlike the SGERTs with a complete and smooth external Au shell (Fig. 1d) ${ }^{2}$, a plenty of nanogaps (indicated by yellow arrows in Fig. 1e) with a size of $1-3 \mathrm{~nm}$ are formed in the rough petal-like structures of the Au shell of P-GERTs, denoted as external nanogaps, which are native electromagnetic hot spots for the Raman enhancement. Thus 4-NBT molecules are further decorated on the external nanogaps via a self-assembly process. The overall diameter of P-GERTs is $66 \pm 4 \mathrm{~nm}$ with a monodispersed particle size and morphology (Fig. 1e).

Aqueous P-GERTs show a dark blue color with a single resonance peak at $600 \mathrm{~nm}$ with a relatively broad linewidth, while aqueous S-GERTs show a typical ruby color with a single pronounced resonance peak at $540 \mathrm{~nm}$ with a much narrower linewidth (Fig. 1f). Compared with S-GERTs, the resonance peak of P-GERTs is redshifted and becomes broadened most likely due to the strong plasmonic coupling of petal-like $\mathrm{Au}$ structures of the rough shell. This also demonstrates a great number of electromagnetic hot spots formed on the external shell of P-GERTs. We then turn to compare the SERS properties of P-GERTs and S-GERTs. It can be seen that the SERS spectrum of P-GERTs when excited by $638 \mathrm{~nm}$ laser exhibits characteristic Raman bands dominated by the strong mode of $v\left(\mathrm{NO}_{2}\right)$ at $1340 \mathrm{~cm}^{-1}$ and four relatively weak modes by $\pi(\mathrm{CH})+\pi(\mathrm{CS})+\pi(\mathrm{CC})$ at $723 \mathrm{~cm}^{-1}, \pi(\mathrm{CH})$ at $854 \mathrm{~cm}^{-1}$, $v(\mathrm{CS})$ at $1083 \mathrm{~cm}^{-1}$, and $v$ (CC) at $1575 \mathrm{~cm}^{-1}$ (Fig. 1g). While S-GERTs exhibit two relatively strong Raman bands at 1055 and $1555 \mathrm{~cm}^{-1}$ and one weak band at $1178 \mathrm{~cm}^{-128,41,42}$. More importantly, we found that the total Raman intensity of PGERTs is more than two orders of magnitude higher than that of S-GERTs under the same experimental condition, estimated by comparing the Raman band at $1340 \mathrm{~cm}^{-1}$ for $4-\mathrm{NBT}$ and at $1055 \mathrm{~cm}^{-1}$ for $1,4-B D T$. It is also found that the Raman intensity of P-GERTs is roughly one order larger than that of the DNA-bridged SERS tags previously reported ${ }^{17}$, after 
a

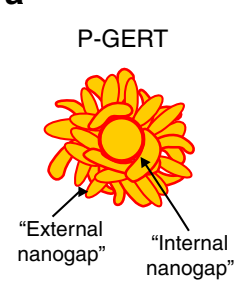

C

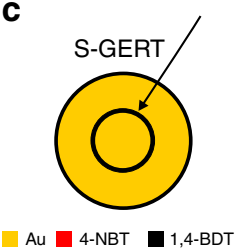

b

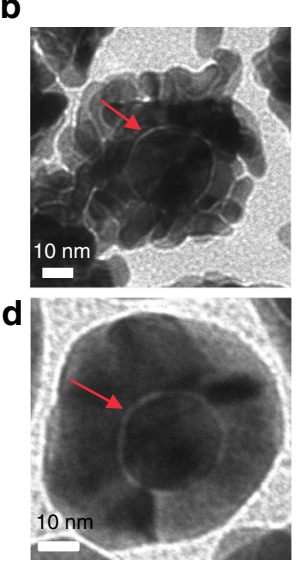

e

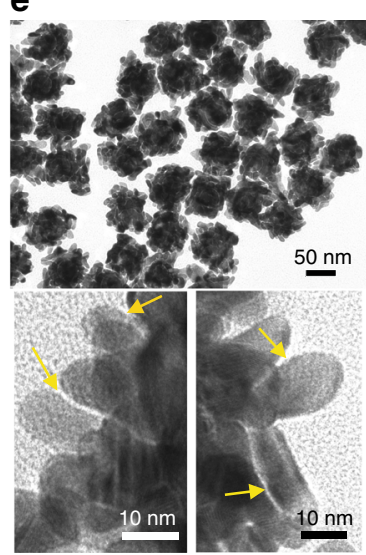

f

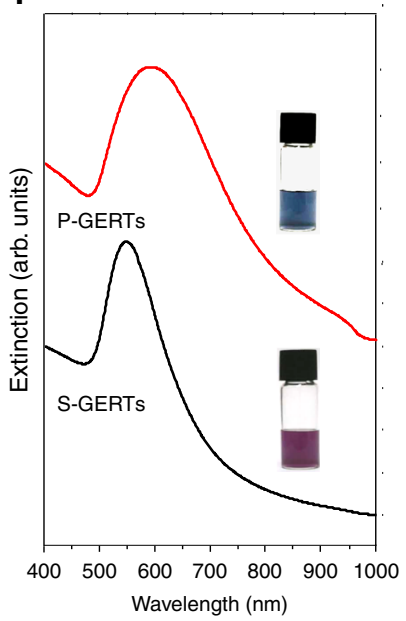

g

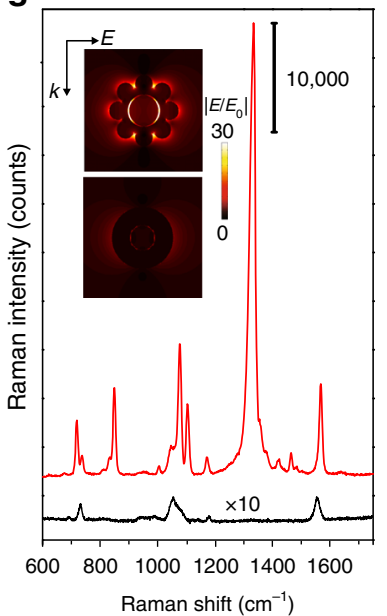

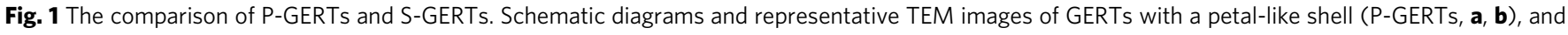
GERTs with a smooth-surface shell (S-GERTs, c, d). Red arrows in panel b, d indicate the internal nanogaps of GERTs. The scale bars are 10 nm. e Representative low-resolution TEM image (top, the scale bar is $50 \mathrm{~nm}$ ) and high-resolution TEM images (bottom, the scale bar is $10 \mathrm{~nm}$ ) of P-GERTs. Yellow arrows in panel e indicate the external nanogaps between petal-like structures on the shell of P-GERTs. $\mathbf{f}$ Extinction spectra of P-GERTs (red) and SGERTs (black). The insets in panel $\mathbf{f}$ show the corresponding photos of GERTs solution. $\mathbf{g}$ SERS spectra of P-GERTs (red) and S-GERTs (black). The insets in panel $\mathbf{g}$ show the finite-difference time-domain (FDTD) calculated electric field enhancement distribution of a single P-GERT (top) and S-GERT (bottom) excited by $638 \mathrm{~nm}$. Raman measurements were performed using $638 \mathrm{~nm}$ laser, $2 \mathrm{~s}$ acquisition time, and $\times 10$ objective lens

normalizing all experimental parameters (see more details in Supplementary Note 4). Different SERS performances can be explained by the finite-difference time-domain (FDTD) calculated electro-magnetic field distributions (insets in Fig. 1g). It can be found that the internal and external nanogaps of the PGERT both produce quite strong electromagnetic field enhancement when excited by $638 \mathrm{~nm}$ laser, which can greatly enhance the Raman signal. But the S-GERT only shows the hot spots in the internal nanogap, and the electromagnetic field enhancement on the external shell is much smaller. We presume that the following three factors have contributed to the ultrahigh Raman intensity of P-GERTs. First, the internal nanogap still exists in P-GERTs, and the plasmon coupling between the $\mathrm{Au}$ core and shell produces strong electromagnetic enhancement in internal nanogap region. The petal-like shell structure of P-GERTs is more conducive to light penetration than the complete shell of S-GERTs, resulting in more efficient light excitation for the internal nanogaps. Second, the larger surface area and multiple external nanogaps in P-GERTs offer a larger number of immobilization site and hot spots for reporter molecules, therefore achieving a strong Raman enhancement ${ }^{43}$. Third, 4-NBT molecules have a larger Raman cross section than 1,4 -BDT due to the intrinsic chemical structure ${ }^{44}$.

To investigate the enhancement contribution of the external hot spots to the total Raman enhancement of P-GERTs, we compared the Raman spectra of P-GERTs before and after the decoration of 4-NBT molecules in the external nanogaps when excited by three different laser wavelengths (532, 638, and $785 \mathrm{~nm}$ ) with a particle concentration of $0.2 \mathrm{nM}$. The $2 \mathrm{~nm}$ redshift of the resonance peak of P-GERTs suggests that the 4NBT molecules adsorbed successfully in the external nanogaps (Supplementary Fig. 1). P-GERTs show remarkable SERS signals for all three excitation wavelengths, and they in general show a trend with the strongest signal at $638 \mathrm{~nm}$, relatively weak at $785 \mathrm{~nm}$, and the weakest at $532 \mathrm{~nm}$ no matter before or after the external decoration of 4-NBT molecules (Supplementary Fig. 2a). Moreover, they all show the Raman intensity improvement after the external decoration of 4-NBT molecules at three wavelengths, for example, $19 \%, 47 \%$, and $21 \%$ for 532,638 , and $785 \mathrm{~nm}$, respectively. In contrast, S-GERTs exhibit much weaker Raman signals under the same three excitation wavelengths, and the functionalization of Raman reporters (1,4-BDT) on the external surface only provides $16 \%, 8.2 \%$, and $10 \%$ intensity improvement for 532, 638, and $785 \mathrm{~nm}$ excitation (Supplementary Fig. 2b). Compared with S-GERTs, the SERS intensities of P-GERTs were around 160-, 128-, and 86-fold higher with the contribution of external hot spots for 532, 638, and $785 \mathrm{~nm}$ laser excitation, respectively (Supplementary Fig. 2c). The SERS enhancement factors (EFs) of P-GERTs are estimated to be $8.7 \times 10^{8}, 5.4 \times 10^{9}$, and $3.7 \times 10^{9}$ for 532,638 , and $785 \mathrm{~nm}$, respectively (see detailed calculations in Methods). These results confirm that the external hot spots play an important role for the Raman enhancement of P-GERTs, and also demonstrate the potential of P-GERTs as multiple-wavelength active SERS probes. The FDTD calculations additionally disclose that the electromagnetic field both inside the internal and external nanogaps of P-GERTs exhibit the strongest enhancement with $638 \mathrm{~nm}$ excitation compared to 532 and $785 \mathrm{~nm}$ excitation (Supplementary Fig. 2d), which is consistent with the experimental results.

Morphology and optical tuning of P-GERTs. In our previous work of S-GERTs ${ }^{45}$, we have found that a longer incubation time of 1,4-BDT reporters with $\mathrm{Au}$ cores can form a multilayered molecular structure, which affects the morphology (e.g., the thickness) of the internal nanogap and the consequent optical properties of GERTs, but their external Au shell remains unaffected with a spherical shape and a smooth surface. Herein, 4NBT molecules were also self-assembled on $\mathrm{Au}$ cores via $\mathrm{Au}-\mathrm{S}$ covalent bonding but cannot form a multilayered molecular structure due to the lack of the second thiol group even when a large excess of 4 -NBT was added ${ }^{45,46}$. After a super-long incubation time of $960 \mathrm{~min}$ for Au cores and 4-NBT, only a $2.6-\mathrm{nm}$ redshift of the plasmon resonance peak was observed (Supplementary Fig. 3), which further confirms that 4-NBT molecules can only form a monolayer on Au cores ${ }^{46}$. However, when we varied the incubation time of the Au cores in 4 -NBT $(10 \mathrm{mM})$ 
solution from 0.5 to $960 \mathrm{~min}$, it exhibited great impact on the surface morphologies and optical properties of P-GERTs. When the incubation time is $0.5 \mathrm{~min}$, the $\mathrm{Au}$ shell is a complete piece with a smooth external surface that is very similar to S-GERTs, but only an incomplete internal nanogap can be observed (Fig. 2a). When the incubation time increases to $2 \mathrm{~min}$, the $\mathrm{Au}$ shell is still intact but with a relatively complete interior nanogap (Fig. 2a). Further increasing of incubation time from 5 to $60 \mathrm{~min}$, the $\mathrm{Au}$ shell starts to become petal-like still with a continuous interior nanogap and multiple external nanogaps (Fig. 2a). The number of petal-like structures increases with the extension of incubation time. After $960 \mathrm{~min}$ incubation, the Au shell is composed of many small petal-like structures, and the identification of interior nanogaps become more difficult (Fig. 2a and Supplementary Fig. 4). TEM measurements of a large number of P-GERTs with various incubation time show that they all have a monodispersed particle size roughly in the range of $60-70 \mathrm{~nm}$ (Supplementary Fig. 5).

To understand how the number of 4-NBT molecules on Au cores influences the growth of $\mathrm{Au}$ shells, we investigated their growth process using time-lapse TEM analysis. Snapshots of intermediate products were obtained by terminating the Au shell growth process at different time points (from 1 to $12 \mathrm{~min}$ ) after 4 -NBT modified $\mathrm{Au}$ cores were introduced into the reaction. When the incubation time is 0.5 and $2 \mathrm{~min}, 4$-NBT exhibits an adsorption density of around 2.7 and 3.3 molecules $/ \mathrm{nm}^{2}$ on $\mathrm{Au}$ cores, according to its molecular footprint of $\sim 0.2 \mathrm{~nm}^{2}$ and the plasmon resonance shifts in Supplementary Fig. $3^{47}$, and they primarily lie down on the $\mathrm{Au}$ core surface and form a leaky monolayer (Fig. 2 b $)^{15}$. The Au shell growth starts with a relatively large nucleation on the core, and gradually turns outward to form the shell and wrap around the $\mathrm{Au}$ core ${ }^{45}$. Since the 4-NBT molecules are in lying-down orientation and the nitro group has a small contact area with the Au shell, the Au shell can easily form a complete piece which is similar to S-GERTs. Further increasing of incubation time to 5-960 $\mathrm{min}$ allows the improvement of the adsorption density to 4-5 molecules $/ \mathrm{nm}^{2}$, and 4-NBT molecules form a more densely packed monolayer on the cores in a more vertical state (Fig. 2c). Due to the poor affinity between Au and the nitro group, the Au shell can only grow from a number of small nucleation sites on cores, and gradually forms petal-like structures, which is more conducive to light penetration ${ }^{31}$. In addition, the internal nanogaps also become more discontinuous. Time-lapse TEM examination offers us a preliminary understanding of the petal-like shell growth mechanism, but a more advanced characterization such as high-resolution in situ TEM may further disclose more insights.

Next, we optimize GERTs for high-speed Raman imaging in terms of far-field extinction spectra and near-field SERS properties for the P-GERTs with different morpholgies after further decoration of 4-NBT molecules into the external nanogaps. Figure $2 \mathrm{~d}$ indicates that the contribution of the Raman enhancement from the internal and external near-field hot spots (shaded area) becomes significant when the incubation time is longer than $2 \mathrm{~min}$ (namely, after forming petal-like shell structures) for both 633 and $785 \mathrm{~nm}$ lasers excitation (see more details in Supplementary Fig. 6). This is obviously due to the formation of near-field hot spots in the internal and external nanogaps. However, S-GERTs with their relatively smooth shells show little signal enhancement effect after adsorbtion of reporter molecules on their shells (Supplementary Fig. 2b, c). This optimization process of the morphology and SERS properties illustrates that P-GERTs with $10 \mathrm{~min}$ incubation time show the optimal Raman intensity, and we proceed in the later experiments using this sample unless otherwise stated. By considering a larger surface area of external shell and a larger molecular Raman cross section, we have also found that the Raman enhancement factor from the external hot spots is roughly one order of magnitude smaller than that from the internal hot spots in P-GERTs although the overall Raman signal is dramatically enlarged. This is probably explained by the difficulty of molecular diffusion into the external nanogaps during the self-assembly process.

Single-NP detection of P-GERTs. Single-NP SERS detection raises new potential for multiplexed molecular diagnosis and in vivo Raman spectroscopy and imaging ${ }^{48-50}$. Therefore, we further challenge the sensitivity and the photostability of P-GERTs down to a single-NP level. Before the measurement, we first coated the P-GERTs with a mesoporous silica layer (MS PGERTs for brevity) to prevent the formation of inter-particle electromagnetic hot spots (Fig. 3a). The MS layer showed a homogeneous thickness of $13 \pm 2.4 \mathrm{~nm}$, which is thick enough to screen the inter-particle plasmon coupling ${ }^{51,52}$. Fig. 3 b shows the representative SERS spectra obtained from aqueous MS P-GERTs with different concentrations of $10 \mathrm{pM}, 1 \mathrm{pM}, 100 \mathrm{fM}, 10 \mathrm{fM}$, and $1 \mathrm{fM}$ excited by $638 \mathrm{~nm}$ laser, indicating clear 4-NBT Raman profiles with a good signal-to-noise ratio even at the lowest concentration of $1 \mathrm{fM}$ (Supplementary Fig. 7). We also notice that we could constantly detect Raman signals from the MS P-GERTs for relatively high concentrations, such as $10 \mathrm{pM}, 1 \mathrm{pM}$, and 100 $\mathrm{fM}$, but only signals in about a quarter of measurements for the concentration of $10 \mathrm{fM}$, and signals in very few cases for the concentration of $1 \mathrm{fM}$. Then we performed Raman measurements in a time sequence with P-GERTs concentration of 100, 10, and 1 $\mathrm{fM}$ in a more accurate way. The probed volume was estimated to be approximately $32 \mathrm{pL}$ according to the method described previously $^{53,54}$, corresponding to an average of $2,0.2$, and 0.02 NP in the probed volume when the concentration is 100,10 , and $1 \mathrm{fM}$, respectively. With such few particles, the average residence time of a particle in the probed volume can be roughly estimated to be 10-20 s due to the Brownian motion into and out of the probed volume ${ }^{53}$, so we set the measurement time as $10 \mathrm{~s}$. Signals measured from a sample without GERTs were used to establish the background threshold of 117 counts, which is three times the standard variation in the mean background signal (indicated by blue dotted lines in Fig. 3d and Supplementary Fig. 8). ${ }^{31}$ Fig. 3c-e display SERS spectra, band intensity and statistical analysis $\left(1340 \mathrm{~cm}^{-1}\right)$ of 120 Raman measurements in a time sequence with an average of $2,0.2$, and $0.02 \mathrm{NP}$ per probed volume. For the case of 2 particles per volume, all 120 measurements exhibited pronounced Raman spectra (Fig. $3 \mathrm{c}$ top) with the band signal $\left(1340 \mathrm{~cm}^{-1}\right)$ beyond the threshold (Fig. $3 \mathrm{~d}$ top), and the corresponding statistical analysis shows a Gaussian statistical distribution of the frequency of the appearance of the Raman signal (Fig. 3e top). When the particle concentration is decreased to an average of 0.2 particles per volume, only $\sim 40 \%$ of measurements meet the threshold criteria (middle in Fig. 3c, d), and the SERS signal distribution exhibits four relative maxima that reflect the probability of finding $0,1,2$ or 3 NPs in the proved volume, respectively. The distribution can be reasonably fit by the superposition of four Gaussian curves, which is roughly consisting with a Poisson distribution (Fig. 3e middle). The results obtained with an average of $0.02 \mathrm{NP}$ per volume further illustrate that only $\sim 3 \%$ measurements show acceptable Raman signals, with three fitted Gaussian curves on the intensity distribution that reflect the probability of finding 0,1 , or 2 NPs in the proved volume. This is roughly consistent with a Poisson distribution as well. When the average number of particles in probed volume is 1 or less, the change in the statistical distribution of SERS signals from Gaussian to Poisson distribution provides evidence for the single-NP detection by SERS ${ }^{53}$. It can be also noticed that the 

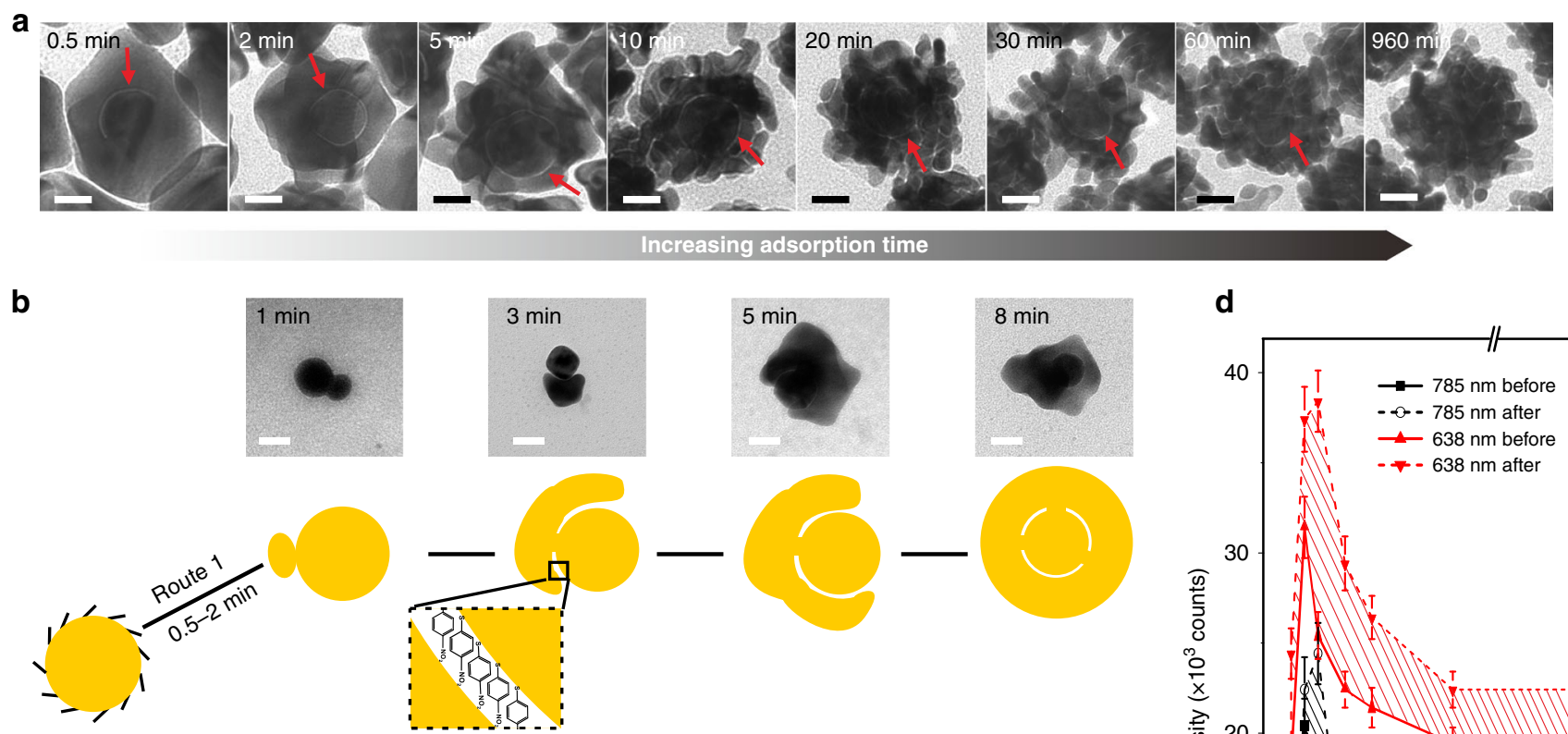

d
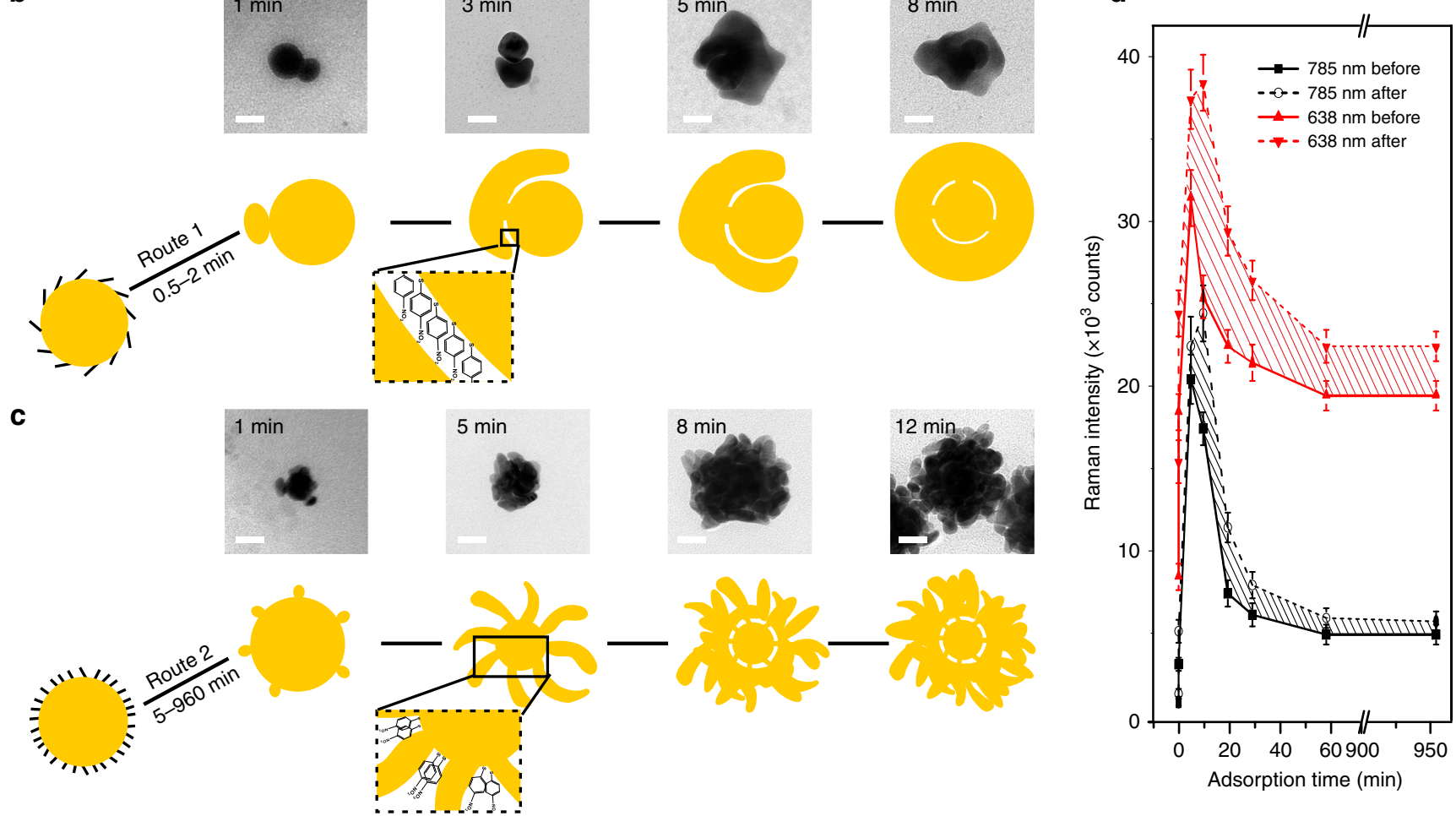

Fig. 2 Highly tunable morphology of P-GERTs. a Representative TEM images of P-GERTs as a function of incubation time (0.5, 2, 5, 10, 20, 30, 60, and 960 min, respectively) of Au core and Raman reporter 4-NBT. Red arrows indicate the internal nanogaps of P-GERTs. Presumed growth process of the Au shell on $\mathbf{b}$ low-density and $\mathbf{c}$ high-density 4-NBT decorated Au cores, with corresponding time-lapse TEM examinations. All scale bars are $20 \mathrm{~nm}$. $\mathbf{d}$ Comparison of mean SERS intensity before and after 4-NBT molecules decoration on the external surface of P-GERTs with various morphologies excited by $785 \mathrm{~nm}$ (black) and $638 \mathrm{~nm}$ (red) laser. Error bars are standard deviations from three independent measurements. Source data are provided as a Source Data file

Raman measurements of ultra-low concentration of aqueous PGERTs excited by $785 \mathrm{~nm}$ show quite similar behaviors to those by $638 \mathrm{~nm}$, only with a slightly lower (around 70\%) Raman intensity (Supplementary Fig. 9). Thus, we conclude the detection sensitivity of P-GERTs down to a single-NP level in liquid state. We have additionally performed the stability analysis of aqueous MS P-GERTs. The extinction and Raman spectra in aqueous solution of different $\mathrm{pH}$ values (from 5 to 10 ) and in $10 \%$ fetal bovine serum (FBS) and saline for $72 \mathrm{~h}$ show stable intensities and unchanged spectral profiles (Supplementary Fig. 10). These results indicate excellent materials stability of aqueous P-GERTs suitable for biomedical applications in different environments.

We further examined the single-NP Raman signal of MS PGERTs in solid state by atomic force microscope (AFM)correlated nano-Raman measurement to quantify their signal uniformity and photostability ${ }^{19}$. It should be noted that we used the dielectric Si tips in the nano-Raman measurement (Fig. 4a), which is obviously distinct from tip-enhanced Raman scattering (TERS) measurement. The MS P-GERTs were coated with a MS layer with a thickness of $13 \mathrm{~nm}$ so that the tip end cannot directly contact with 4-NBT molecules. NP solution was first spin-coated on a silicon wafer and dried, and then the laser focal spot was exactly matched with the center of the AFM $\mathrm{Si}$ tip for symmetrical scattering on the Si tip end. The Raman measurement could be performed in a contact or non-contact mode. A typical non-contact mode AFM image gives a confirmation of the formation of isolated single NPs on the substrate with a particle height of $\sim 96 \mathrm{~nm}$ (Fig. 4b). Figure 4c displays the correlated SERS spectra from the corresponding three particles (particle 1-3) in Fig. $4 \mathrm{~b}$ measured in contact and non-contact modes. These three particles all show very strong and uniform Raman signals of 4NBT molecules ( 450 counts) in the contact mode, which is about $10 \%$ stronger than that in the non-contact mode. This may be because the tip can assist the focus of light better onto the particles in the contact mode. More than $80 \%$ of 34 single-NP measurements produced Raman signals between 350 and 450 counts, demonstrating a quite good uniformity at the single-NP level (Fig. 4d). Although the petal-like structures of P-GERTs are random and poorly controlled, each P-GERT has a large number of petal-like structures on the surface, which results in an average Raman signal of each NP in a decent uniformity. More importantly, single-NP photostability examinations of P-GERTs 
a
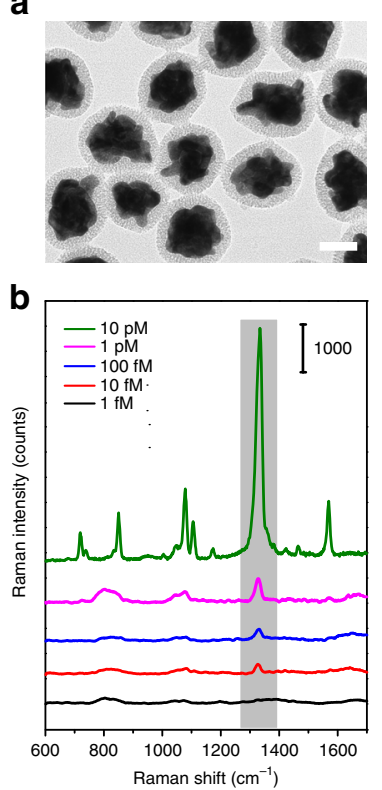

C
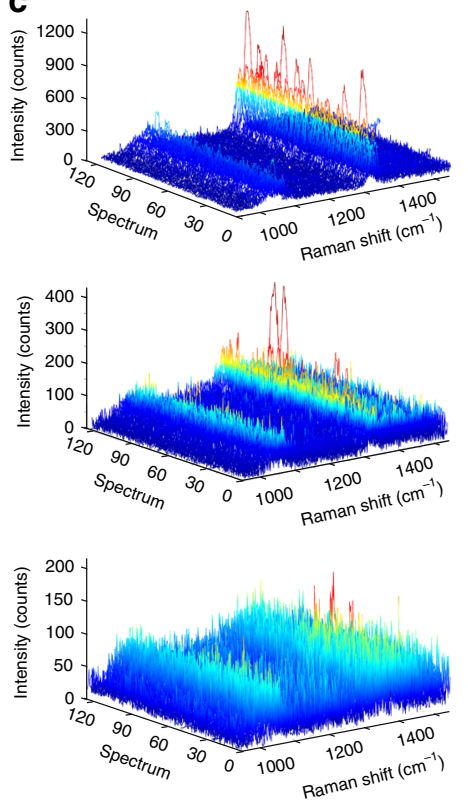

d
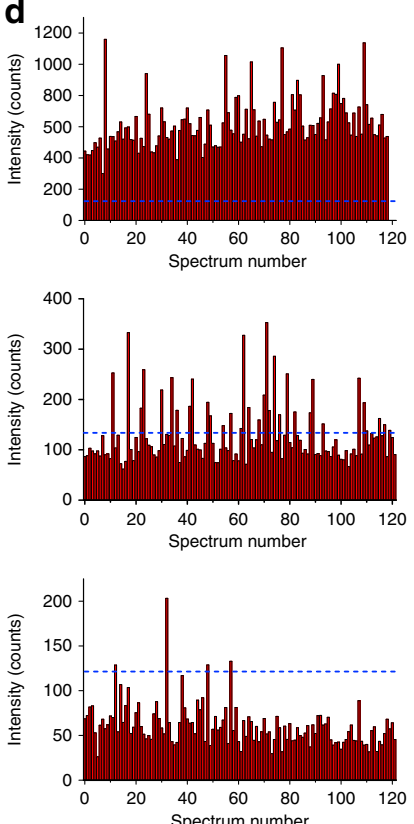
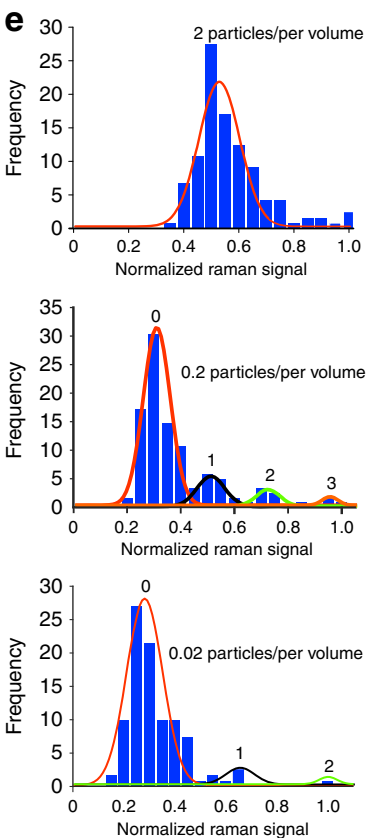

Fig. 3 Single-NP detection of aqueous P-GERT solution. a TEM image of mesoporous silica-coated (MS) P-GERTs. Scale bar is $20 \mathrm{~nm}$. b Concentrationdependent Raman measurements of aqueous MS P-GERTs ( $638 \mathrm{~nm}$ laser, $20 \mathrm{~mW}, 10 \mathrm{~s}$ acquisition time, and $\times 60$ objective lens). c SERS spectra plot, $\mathbf{d}$ the intensity of Raman band at $1340 \mathrm{~cm}^{-1}$, and e the corresponding statistical analysis of 120 measurements from 100 (top), 10 (middle), and 1 (bottom) fM PGERT solution. 100, 10, and $1 \mathrm{fM}$ GERT solutions correspond to 2, 0.2 , and 0.02 particle per probing volume, respectively. Blue dotted lines in panel d represent the background Raman signal $\left(1340 \mathrm{~cm}^{-1}\right)$ without P-GERTs. All solid curves in panel e are Gaussian fitting to the data. The fitted peaks show the Gaussian (top) or Poisson (middle and bottom) distribution of the data. For Poisson distribution, the red, black, green and orange peaks reflect the probability to find $0,1,2$, or 3 particles per volume

indicate that their SERS signals remain quite stable after continuous laser irradiation $(110 \mu \mathrm{W})$ for $1800 \mathrm{~s}$ (Fig. 4e). The inset in Fig. 4e shows all time-resolved spectra of a single NP during continuous irradiation without significant fluctuation or appearance of new Raman bands. Their super-stable SERS properties are further proved by the almost negligible change of the three representative SERS spectra measured before, during, and after irradiation (Fig. 4f). All these results imply that PGERTs exhibit a large Raman enhancement down to a single-NP detection sensitivity, decent particle-to-particle SERS uniformity, and ultrahigh photostability.

High-speed and high-contrast Raman cell and tissue imaging. It typically takes minutes to hours to acquire a Raman cell image with SERS tags 55,56 . We have recently demonstrated that SGERTs-based cell imaging with a resolution of $50 \times 50$ pixels could be obtained within $35 \mathrm{~s}$ with a much higher laser power of $3.7 \mathrm{~mW}^{30}$. Such large Raman enhancement of P-GERTs down to a single-particle level may further allows us to improve the imaging speed with a further lower laser power, which is crucial to minimize the photodamage to biological samples. We performed a high-speed Raman cell imaging in a step-by-step mapping mode, where the laser is scanned across the cell in $X$ and $Y$ directions by the rotation of galvo mirrors instead of moving the stage mechanically (see Fig. 5a). As a demonstration, Fig. $5 b-d$ show the bright-field image, Raman image and overlay of a fixed H1299 cell passively stained with the P-GERTs. The brightfield image and Raman image of the cell coincided well. The highresolution single-cell Raman image $(50 \times 50$ pixels for $55 \times$ $66 \mu \mathrm{m}^{2}$ field of view) could be acquired within $6 \mathrm{~s}$ with a laser power $370 \mu \mathrm{W}$ and an ultrashort acquisition time of $0.7 \mathrm{~ms}$ per pixel (see more details in Methods). $0.7 \mathrm{~ms}$ is so far the shortest acquisition time of commercial electron-multiplying charge- coupled device (EMCCD) to the best of our knowledge. It allows to largely improve the imaging speed one to two orders of magnitude faster compared to the previous work performed with a similar image resolution and laser power ${ }^{4}$. Raman imaging of multiple cells in a larger area $\left(61 \times 41\right.$ pixels for $380 \times 240 \mu \mathrm{m}^{2}$ field of view) can also be accomplished within $7 \mathrm{~s}$ (Supplementary Fig. 11). Such high imaging speed can be attributed to the significant improvements in SERS tag signal, imaging system, and data processing method. Firstly, P-GERTs with multiple electromagnetic hot spots can provide sufficient Raman signals, which greatly shorten the signal acquisition time. Secondly, the step-bystep imaging mode realizes rapid movement of the laser spot by the rotation of galvo mirrors, which greatly reduces the scanning time. Thirdly, data processing line-by-line in parallel in SWIFT mode also helps time saving ${ }^{57-59}$. More importantly, three Raman spectra randomly selected from different parts (point 1-3 in Fig. 5c) of the cell clearly exhibit characteristic bands of 4-NBT molecules with a rather good signal-to-noise ratio within such an ultrashort acquisition time of $0.7 \mathrm{~ms}$ per pixel (Fig. 5e). These result in the possibility to detect a single cell labelled with less than 100 P-GERTs based on the aforementioned single-NP AFM nano-Raman measurements (refer to Supplementary Fig. 12 for more details). In addition, the background Raman and autofluorescence signals from the cell and the culture dish are negligible within such a short exposure time, consequently resulting in an ultrahigh-contrast Raman image (Fig. 5c). It can be also seen from Fig. 5b that the addition of nanoprobes did not generate a significant effect on the morphology of cell, indicating P-GERTs may have good biocompatibility. A CCK-8 assay test further proved that the P-GERTs are nearly non-cytotoxic to cells within a concentration range of $0-1 \mathrm{nM}$ (Supplementary Fig. 13), implying great potential for in vitro and in vivo bioimaging. We additionally demonstrated that P-GERTs exhibited excellent 
a

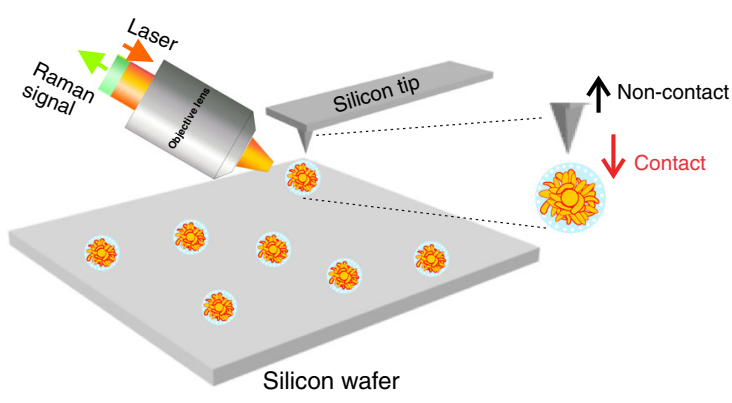

b

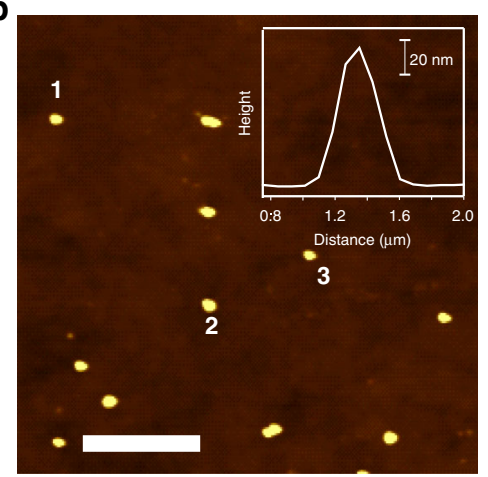

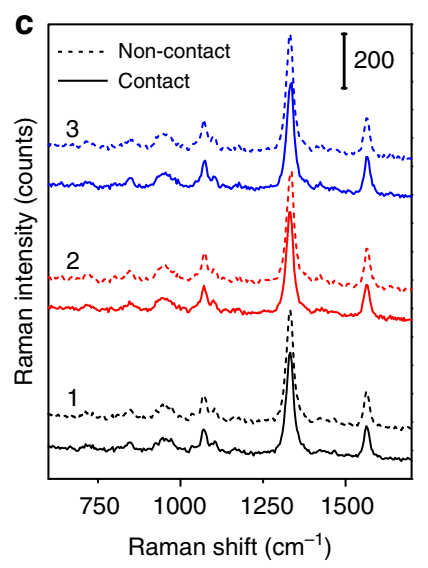
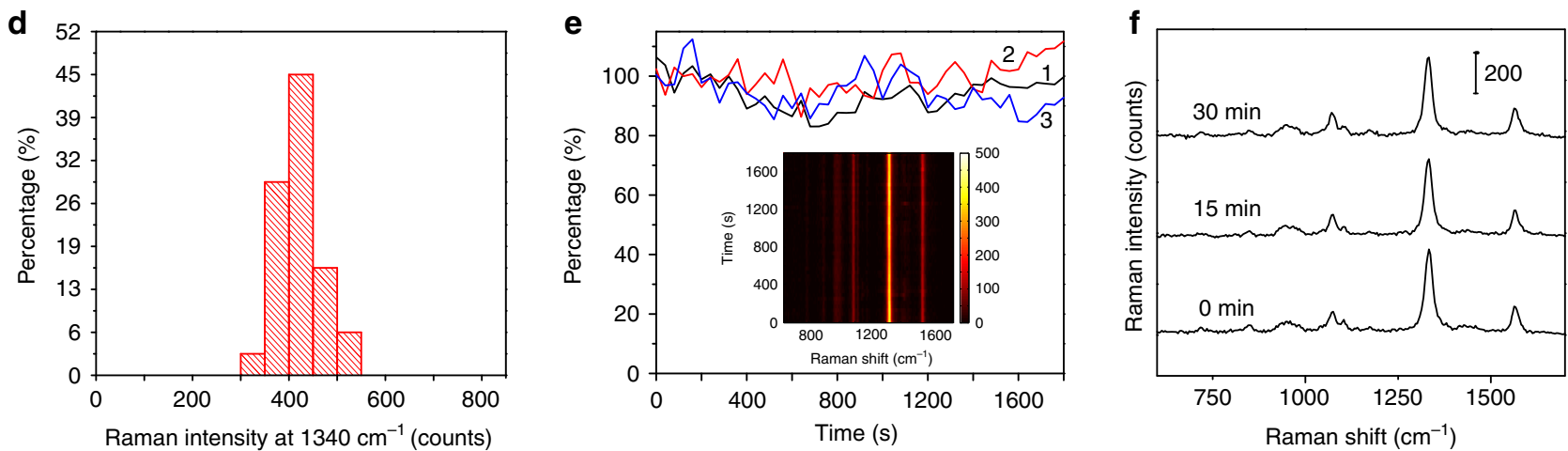

Fig. 4 AFM-correlated single-NP nano-Raman analysis of the P-GERTs. a Schematic illustration of AFM-correlated nano-Raman spectroscopy. b A representative AFM image of MS P-GERTs on silicon wafer. The scale bar is $5 \mu \mathrm{m}$. The inset shows a typical cross-section profile of a single MS P-GERT. c SERS spectra of three single MS GERTs (particle 1, 2, and 3) indicated in panel $\mathbf{b}$ measured in contact and non-contact modes. $\mathbf{d}$ Distribution diagram of the SERS intensity $\left(1340 \mathrm{~cm}^{-1}\right)$ of thirty-four single MS GERTs from different areas on silicon wafer. e Photostability measurements of three single MS GERTs under continuous laser irradiation for $30 \mathrm{~min}$, monitored by the integrated band area at $1340 \mathrm{~cm}^{-1}$. The inset shows a typical time-resolved SERS spectral map of a single GERT during continuous irradiation for $30 \mathrm{~min}$. f Three representative SERS spectra at selected irradiation times under irradiation from the same particle. All measurements were performed with a $633 \mathrm{~nm}$ laser, $110 \mu \mathrm{W}$ power, $10 \mathrm{~s}$ acquisition time, and $\times 100$ objective lens

long-term photostability during high-speed 30-min continuous cell imaging process (Supplementary Fig. 14) and the great potential for high-speed live-cell imaging (Supplementary Fig. 15). Therefore, P-GERTs provide various advantages for Raman cell imaging such as decreased incident laser power, high spatial and temporal resolution, high-contrast image quality, and longterm photostability.

The sentinel lymph nodes (SLNs) are the theoretical first site of cancer metastasis, and the accurate locating of SLNs is fundamental for performing sentinel lymph node biopsy (SLNB) to determine lymphatic spread of cancer cells. To demonstrate the potential of P-GERTs for in vivo imaging, we performed Raman imaging of the SLN in mice. The mice $(n=3)$ were injected subcutaneously with P-GERTs in saline $(25 \mu \mathrm{L}, 0.5 \mathrm{mg} / \mathrm{mL})$ in the paw of the left hind limb and the popliteal lymph node was modeled as the SLN. After $24 \mathrm{~h}$, the mice were anesthetized and high-speed Raman imaging of SLNs were performed in a Macro mapping mode (see Fig. 5f), where the laser beam is rasterscanned to record an average Raman spectrum across an area sub-millimeter by sub-millimeter and the sample is moved by the motorized XY stage with a step to cover the whole surface. Compared to the traditional motorized stage movement with a normal laser spot (typically several micrometers in diameter), we can minimize the time spent on stage response and movement as much as possible without losing effective Raman information in the Macro mapping mode. Raman images in Fig. $5 \mathrm{~g}$, $\mathrm{h}$ show strong SERS signals of P-GERTs from the SLN and the injection site, while negligible signal from the surrounding tissues, indicating that P-GERTs can be specifically enriched in the SLNs by lymphatic circulation to trace SLNs. The wide-area in vivo Raman image $\left(3.2 \times 2.8 \mathrm{~cm}^{2}\right)$ was obtained within $52 \mathrm{~s}(20 \times 20$ pixels) with a laser power of $370 \mu \mathrm{W}$ and $0.7 \mathrm{~ms}$ acquisition time per pixel. During the SLN imaging process, it is worth noting that each pixel contains all Raman signals over an area of $600 \times$ $600 \mu \mathrm{m}^{2}$, which usually requires hundreds of seconds in normal stage movement mode. The Macro mapping mode enables us to collect sample information as much as possible with a short imaging time and few pixels.

The Raman spectra from SLN (point 2), adjacent tissues (point 1) and the surrounding environment (point 3) further confirms the specificity of P-GERTs for lymph node tracing (inset in Fig. 5h). Because the signal acquisition time is only $0.7 \mathrm{~ms}$, the signal generated by tissue is not obvious and similar to the blank background. Figure $5 \mathrm{i}$ shows cross sections of the Raman signal intensity $\left(1340 \mathrm{~cm}^{-1}\right)$ and the signal-to-background ratio (SBR) from the Raman image, marked by a white dashed line in panel $\mathrm{h}$ of Fig. 5h. The Raman signal of the surrounding tissues is considered as the background. Although the acquisition time per pixel is only $0.7 \mathrm{~ms}$, the Raman signal acquired in the SLN region reaches a SBR of around 6.5. Unlike fluorescent nanoprobes, PGERTs have a unique fingerprint Raman signal from 4-NBT molecules, which is not available from biological tissues. Therefore the raw Raman spectrum (for example, point 2 in the inset of panel h) can be further processed to remove the background 
a

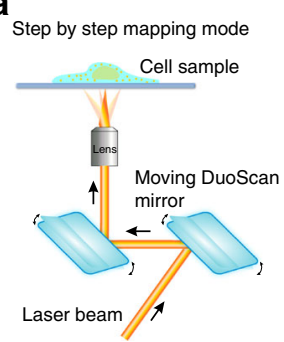

f

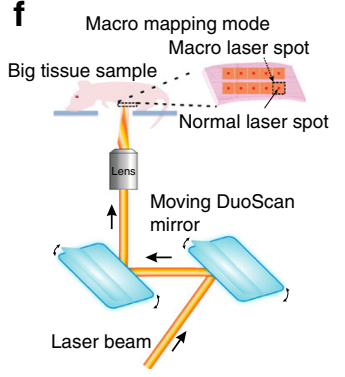

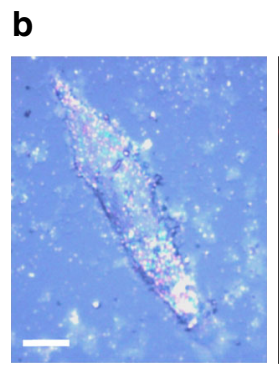
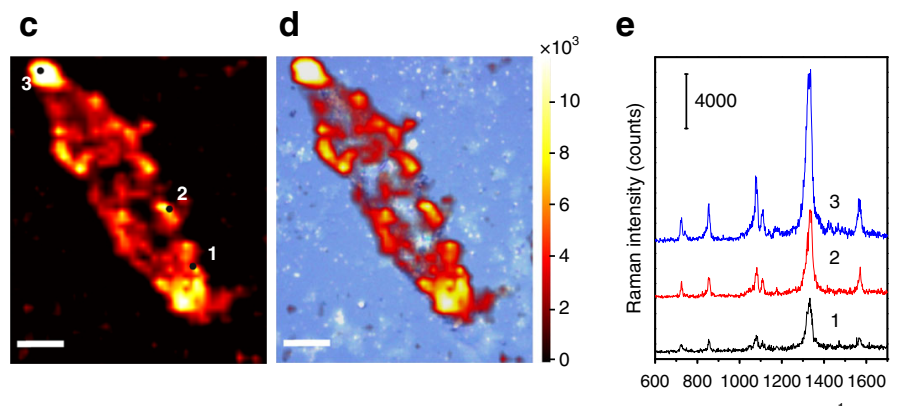

g

h

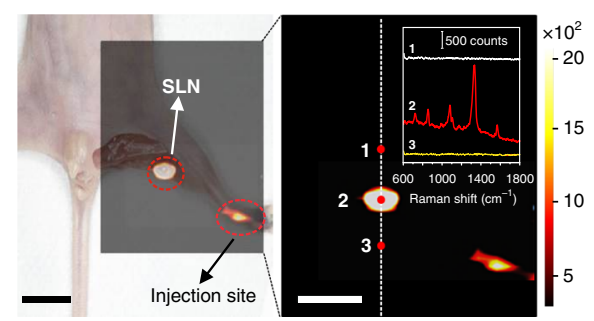

i

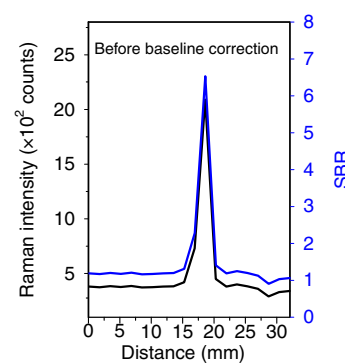

j

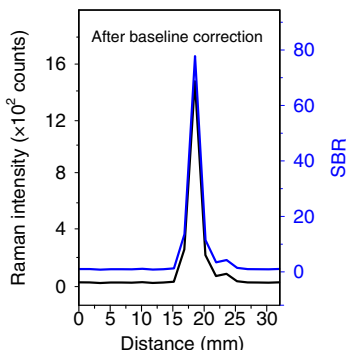

Fig. 5 High-speed and high-contrast wide-area Raman imaging. a Schematic illustration of the stepping scan mode for high-speed cell Raman imaging.

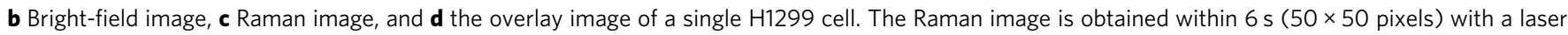

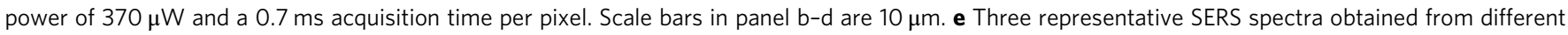
points in panel c. f Schematic illustration of the Macro scan mode for wide-area tissue imaging. (g, h) High-contrast and wide-area in vivo Raman image

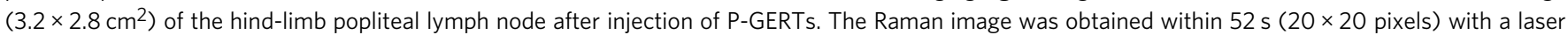
power of $370 \mu \mathrm{W}$ and acquisition time of $0.7 \mathrm{~ms}$ per pixel. Scale bars in panel $\mathbf{g}$ and $\mathbf{h}$ are $1 \mathrm{~cm}$. The inset in $\mathbf{h}$ shows Raman spectra at the SLN in situ (point 2), adjacent tissue (point 1) and surrounding environment (point 3). All Raman images are plotted using the Raman band at $1340 \mathrm{~cm}{ }^{-1}$. The intensity and signal-to-background ratio (SBR) of the Raman signal $\left(1340 \mathrm{~cm}^{-1}\right)$ cross-section from top to bottom marked by the white dashed line in $\mathbf{h}$ (i) before and $\mathbf{j}$ after the baseline correction of Raman spectra

signals such as autofluorescence from adjacent tissues. The SBR of the Raman in vivo imaging can be greatly improved by an order of magnitude to around 80 (see Fig. 5j) after the baseline correction of the raw Raman spectra, realizing extremely highcontrast in vivo imaging. The enrichment of P-GERTs in the SLN was also confirmed by imaging a dissected lymph node (Supplementary Fig. 16). With the advantageous features including strong Raman enhancement, unique fingerprint Raman signal, high photostability, and good biocompatibility, P-GERTs hold the great potential to be used for high-speed and highcontrast intraoperative wide-area Raman imaging.

Multiplexed Raman cell imaging. By replacing Raman reporters immobilized in the external nanogaps of P-GERTs, we can further demonstrate their multiplexing imaging capability. We introduced four kinds of molecules including 3,4-dichlorobenzenethiol (3,4-DBT), 4-mercaptobenzonitrile (4-MBN), 3fluorothiophenol (3-FTP), and 2-naphthalenethiol (2-NT) into the external nanogaps of the petal-like shell of P-GERTs (Fig. 6a). These four kinds of molecules are chosen because they all have a thiol group, forming a covalent bonding to the Au surface of P-GERTs, and each molecule has at least one characteristic Raman band that can be better distinguished from 4-NBT molecule. We obtained Raman spectra of P-GERTs with these four kinds of reporters with different characteristic bands (Fig. $6 \mathrm{~b})$, indicating the unique characteristic band of $2230 \mathrm{~cm}^{-1}$ for 4 MBN (purple), $1001 \mathrm{~cm}^{-1}$ for 3-FTP (green), $568 \mathrm{~cm}^{-1}$ for 3,4DBT (red), and $1379 \mathrm{~cm}^{-1}$ for 2-NT (blue). The cells were incubated with the mixture of four kinds of GERTs (1:1:1:1 for molar concentration) for $6 \mathrm{~h}$, and then multiplexed Raman imaging was performed. A multiplexed Raman image of a single cell $\left(50 \times 50\right.$ pixels for $24 \times 66 \mu \mathrm{m}^{2}$ field of view) could be acquired within $43 \mathrm{~s}$ with a laser power of $370 \mu \mathrm{W}$ and $10 \mathrm{~ms}$ acquisition time per pixel. As shown in Fig. $6 c$, d, the Raman cell image reconstructed from the characteristic band of $1340 \mathrm{~cm}^{-1}$ of 4-NBT, the common reporters embedded in the internal nanogaps of all four kinds of GERTs, were well consistent with the bright-field image. The Raman images reconstructed using the unique characteristic bands of four external reporters clearly show the slightly different distribution of each kind of GERTs in the cell (Fig. 6e). The multiplexed encoding ability of P-GERTs makes it potential for imaging nanoprobes targeting to different sites of cells or different cells.

\section{Discussion}

In summary, we have developed P-GERTs with 4-NBT Raman reporters embedded in electromagnetic hot spots from the interior nanogaps in the core-shell junction and external nanogaps in the petal-like shell structure for high-speed, high-contrast cell and tissue bioimaging. P-GERTs exhibit around two orders of magnitude stronger SERS signals than S-GERTs at all three laser excitation wavelengths of 532, 638, and $785 \mathrm{~nm}$, reaching a maximum Raman EF beyond $5 \times 10^{9}$. The change in the statistical distribution of SERS signals from Gaussian to Poisson distribution from continuously decreased concentration of aqueous PGERTs demonstrates a detection sensitivity of the single-NP level. The nano-Raman measurements confirm the single-NP sensitivity in solid state and additionally show decent NP uniformity and photostability, evaluated at a single-NP level. The high SERS signal improvements of P-GERTs are attributed to the larger 
a

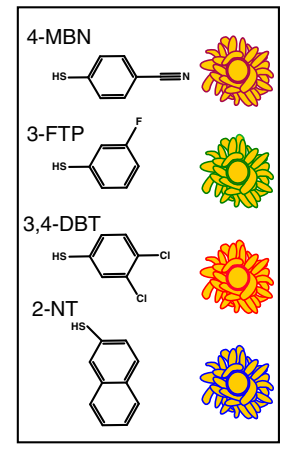

b

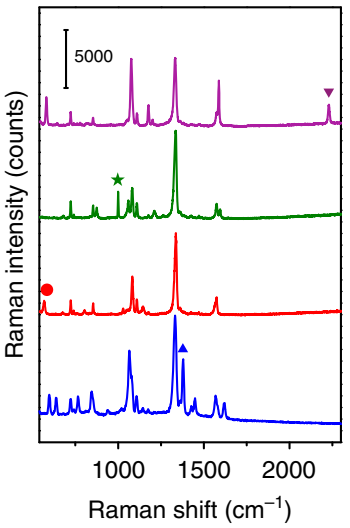

Raman shift $\left(\mathrm{cm}^{-1}\right)$
C

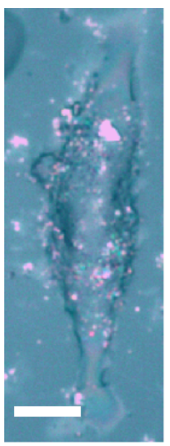

d

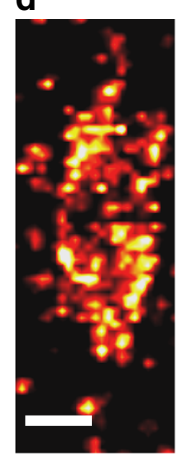

e

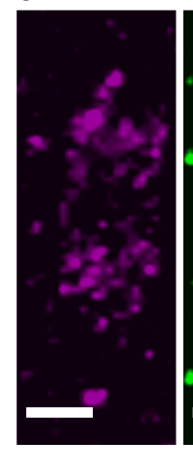

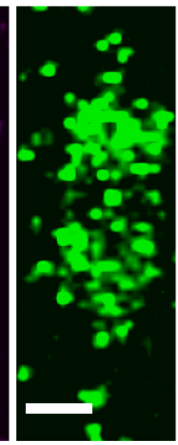

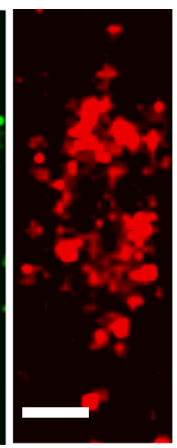

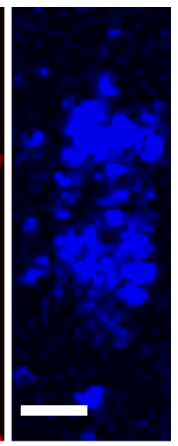

Fig. 6 Multiplexed Raman imaging of a H1299 cell. a Schematics and b SERS spectra of P-GERTs with different Raman reporters of 4-MBN (purple), 3-FTP (green), 3,4-DBT (red), and 2-NT (blue) immobilized in the external nanogaps. c Bright-field image and (d, e) multiplexed SERS cell imaging of a single H1299 cell. Raman image in panel d was plotted from $1340 \mathrm{~cm}^{-1}$ of embedded 4-NBT reporter and images in e were plotted from the Raman signals from external Raman reporters 4-MBN (purple), 3-FTP (green), 3,4-DBT (red), and 2-NT (blue), respectively. All scale bars are $10 \mu \mathrm{m}$

surface area for molecular decoration, large Raman scattering cross-section of 4 -NBT molecules and the difference in shell morphology, where S-GERTs are in a complete piece with a smooth surface while P-GERTs have a petal-like structure with multiple nanogaps. By adjusting the amount of 4-NBT adsorbed on $\mathrm{Au}$ cores, we can regulate the morphology and SERS performance of P-GERTs. By changing the type of external Raman reporters, a variety of probes can be obtained to achieve multiplexed detection and imaging. Due to the ultra-strong Raman signal of P-GERTs, ultra-fast laser scanning technique and data processing method, we have achieved high-speed high-resolution single-cell Raman imaging $\left(50 \times 50\right.$ pixels for $55 \times 66 \mu \mathrm{m}^{2}$ field of view) acquired within $6 \mathrm{~s}$ with a laser power $370 \mu \mathrm{W}$ and acquisition time of $0.7 \mathrm{~ms} /$ pixel, and high-contrast wide-area in vivo SLN Raman imaging $\left(3.2 \times 2.8 \mathrm{~cm}^{2}\right)$ obtained within $52 \mathrm{~s}$ with a laser power of $370 \mu \mathrm{W}$, acquisition time of $0.7 \mathrm{~ms} /$ pixel, and the SBR of around 80. P-GERTs may open new opportunities for rapid ultrasensitive biosensing and bioimaging platform as superbright and highly stable optical nanolabels.

\section{Methods \\ Materials. All materials were used as received without any further purification. Chloroauric chloride $\left(\mathrm{HAuCl}_{4} \cdot 4 \mathrm{H}_{2} \mathrm{O}\right)$, ascorbic acid, tetraethyl orthosilicate (TEOS), sodium hydroxide $(\mathrm{NaOH})$, and methanol were purchased from Sino- pharm Chemical Reagent Co. Ltd (Shanghai, China). Sodium borohydride $\left(\mathrm{NaBH}_{4}\right.$, 98\%), cetyltrimethylammonium chloride (CTAC, 99\%), 3, 4-dichlorobenzenethiol (3,4-DBT, 98\%), 2-naphthalenethiol (2-NT, 98\%), 3-fluorothiophenol (3-FTP, $98 \%$ ) and 4-mercaptobenzonitrile (4-MBN, 95\%) were obtained from J\&K Che- mical Ltd (Shanghai, China). 4-Nitrothiophenol (4-NBT, 90\%) was purchased from Fluorochem Ltd (Derbyshire, United Kingdom). 1,4-Benzenedithiol (1,4-BDT, 98\%) was received from TCI (Tokyo, Japan). The ultra-fine insulin syringes $(1 \mathrm{~mL})$ were purchased from Becton, Dickinson and Company (USA). The cell counting kit-8 (CCK-8) was purchased from Dojindo Laboratories (Kumamoto, Japan). DMEM/High glucose medium, penicillin-streptomycin mixture solution, fetal bovine serum (FBS) and $0.25 \%$ trypsin-EDTA were purchased from Gibco Life Technologies (USA). Nanopure water $(18.2 \mathrm{M} \Omega$ ) was used for all experiments.}

Synthesis of P-GERTs and S-GERTs. Au cores were first synthesized using a seedmediated process described in our previous work ${ }^{28}$. Next, $500 \mu \mathrm{L}$ of $10 \mathrm{mM}$ 4-NBT ethanol solution was added to $\mathrm{Au}$ cores $(10 \mathrm{~mL})$ under vigorous sonication. The mixtures were incubated at $25^{\circ} \mathrm{C}$ for $0.5,2,5,10,20,30,60$, and $960 \mathrm{~min}$, respectively. These 4 -NBT modified cores were then resuspended in $0.05 \mathrm{M} \mathrm{CTAC}$ after washing three times with $0.05 \mathrm{M} \mathrm{CTAC}$ solution. After that, various incubation time of $1 \mathrm{~mL} 4$-NBT modified core solution were added into the mixed growth solution of $16 \mathrm{~mL}$ CTAC solution $(0.05 \mathrm{M}), 480 \mu \mathrm{L}$ of ascorbic acid $(0.04 \mathrm{M})$, and $960 \mu \mathrm{L}$ of $\mathrm{HAuCl}_{4}(4.86 \mathrm{mM})$ under vigorous sonication. The color of the solution changed from colorless to pink, purple, and blue, and 4-NBT GERTs (P-GERTs) can be obtained in about 10 min. 1,4-BDT GERTs (S-GERTs) were synthesized according to the procedure described in our previous work ${ }^{28}$.
Mesoporous silica coating of 4-NBT GERTs was applied according to Gorelikov and Matsuura's protocol with some modifications ${ }^{60}$. The obtained 4-NBT GERTs $(50 \mathrm{~mL})$ were washed three times and then dispersed in $5 \mathrm{~mL}$ water. $\mathrm{NaOH}$ solution $(40 \mu \mathrm{L}, 0.1 \mathrm{M})$ was added with stirring. Three $50 \mu \mathrm{L}$ injections of $5 \%$ TEOS in methanol were carried under gentle stirring at $30 \mathrm{~min}$ intervals, and the reaction was kept for $24 \mathrm{~h}$. The resulting products were collected by centrifugation and washed with ethanol $(25 \mathrm{~mL})$ containing $\mathrm{NH}_{4} \mathrm{NO}_{3}(10 \mathrm{mg})$ for three times.

Modification of Raman reporters on external nanogaps of P-GERTs. The obtained 4-NBT GERTs $(10 \mathrm{~mL})$ were mixed with $500 \mu \mathrm{L}$ of $10 \mathrm{mM}$ Raman reporters (4-NBT, 4-MBN, 3-FTP, 3,4-DBT, 2-NT, respectively) under vigorous sonication for $1 \mathrm{~h}$. The samples were then washed for three times by centrifuge at $11000 \mathrm{rpm}$ for $10 \mathrm{~min}$ to remove excess reagent, and further dispersed in $5 \mathrm{~mL}$ aqueous CTAC solution $(0.05 \mathrm{M})$. Mesoporous silica coating was then applied to the obtained samples.

Characterization of GERTs. Transmission electron microscopy (TEM) images were collected on a JEM-2100F TEM (JEOL, Tokyo, Japan) operated at $200 \mathrm{kV}$ UV-Vis spectra were obtained using a UV1900 UV-Vis spectrophotometer (Aucybest, China). The Raman test was carried out on confocal Raman microscope with 532, 638 and $785 \mathrm{~nm}$ laser (Horiba, Xplora INV). The $638 \mathrm{~nm}$ laser provided by Horiba was an air cooled CLDS laser diode with a linewidth smaller than $0.03 \mathrm{~nm}$. Nano-Raman measurements were carried out on atomic force microscope (AFM)-correlated Raman system $(633 \mathrm{~nm}, \times 100$ objective, NA =0.9). An enzymelinked immunosorbent assay microplate reader (Synergy 2, Bio-TEK) was used to measure the absorbance. Time-lapse TEM analysis of intermediate products were obtained by terminating the Au shell growth process at different time points (from 1 to $12 \mathrm{~min}$ ) after 4 -NBT modified Au cores were introduced into the reaction. Typically, a holey carbon-coated copper grid was immersed into the reaction mixture and immediately put into liquid nitrogen to stop the reaction. The copper grid with specimens attached was used for further TEM characterizations.

FDTD simulation. All the optical simulations were performed with the commercial software FDTD Solutions (Lumerical Inc., Canada). The empirical permittivity of $\mathrm{Au}^{61}$ was fitted with the multi-coefficient model $(\mathrm{MCM})^{62}$. The refractive index of self-assembly 4 -NBT monolayer was set as 1 according to other literatures ${ }^{63,64}$. The surrounding medium was set as water which has the refractive index of 1.33 . The refined mesh area with a smallest mesh size of $0.1 \mathrm{~nm}$ was utilized to cover the whole structure of GERT thus the accuracy of simulation can be guaranteed. The detailed 3D model of the P-GERT was described in the Supplementary Fig. 17.

The limit of detection for P-GERTs in liquid. The limit of detection for P-GERTs was determined by analyzing solutions at various concentrations from $10^{-11}$ to $10^{-15} \mathrm{M}$ using $\times 10(\mathrm{NA}=0.3), 30 \mathrm{~mW}$ laser power and $10 \mathrm{~s}$ integration time. Then, 120 SERS spectra of homogenous solutions of P-GERTs were collected at various concentrations for data analysis.

SERS enhancement factor calculation. A silicon wafer was used for signal calibration during all the Raman measurements. The enhancement factor is a typical indicator for evaluating the performance of SERS nanoprobes. To evaluate the enhancement effect of P-GERTs and compare them to S-GERTs, we performed enhancement factors (EF) calculation of P-GERTs with various Au core-NBT 
incubation time from 0.5 to $960 \mathrm{~min}$. First, the SERS intensity and the ordinary Raman intensity need to be normalized to the number of excited molecules, and then the ratio of the two is calculated to obtain EF:65

$$
\mathrm{EF}=\frac{I_{\text {SERS }} / N_{\text {SERS }}}{I_{\text {Raman }}} / N_{\text {Raman }}
$$

For normal Raman measurements, the concentration of Raman molecule was $1 \mathrm{mM}$. To calculate the concentration of SERS active molecules on each probe, the surface area of gold nanospheres with a diameter of $20 \mathrm{~nm}$ is about $1256 \mathrm{~nm}^{2}$; assuming a $0.2 \mathrm{~nm}^{2}$ footprint for each Raman molecule 47,66 , we evaluate the amount of Raman molecules to be 6280 per P-GERT. Note that this is a maximal amount of SERS active molecules. The use of this value in further calculations gives a minimal estimation for $\mathrm{EF}$.

\section{AFM-correlated single-particle nano-Raman detection and photostability test} AFM-correlated Nano Raman system (AIST, Horiba) was used for single-particle detection after the sample (1 pM) was dried on a silicon wafer. First, AFM was used to find the monodispersed particles on the silicon wafer, then the laser spot was coupled to the tip of the silicon probe to collect single-particle Raman signal (633 nm laser, $\times 100$ objective, $110 \mu \mathrm{W}$ and $10 \mathrm{~s}$ integration time). Particles with a distance more than $2 \mu \mathrm{m}$ from each other were selected for testing and excluding inter-particle coupling effect. Thirty-four single P-GERTs at different regions were tested and then the Raman signal intensity of the single particles was counted. In addition, we performed the photostability of Raman signal of single P-GERT by continuous irradiation for $30 \mathrm{~min}$ under the same conditions, and then the variation of Raman signal was analyzed.

Cell culture. The human lung cancer cell line H1229 was obtained from American Type Culture Collection (ATCC), and all reagents for the cell culture were purchased from Gibco. The cells were cultured in RPMI 1640 medium supplemented with $10 \%$ fetal bovine serum, $100 \mathrm{U} \mathrm{mL}^{-1}$ penicillin, and $100 \mathrm{mg} \mathrm{mL}^{-1}$ streptomycin at $37^{\circ} \mathrm{C}$ in a humidified atmosphere containing $5 \% \mathrm{CO}_{2}$.

Cell proliferation assay. For evaluating the cytotoxicity of P-GERTs on cells, we investigated the effects of P-GERTs on H1299 cells by using CCK-8 assay (Cell Counting Kit-8, Dojindo). The H1299 were seeded on 96-well plates at a density of $3 \times 10^{3}$ cells per well, and cultured in RPMI 1640 medium at $37^{\circ} \mathrm{C}$ in a humidified atmosphere containing $5 \% \mathrm{CO}_{2}$. After $12 \mathrm{~h}$, the culture medium was refreshed and P-GERTs were added at final concentrations of $0.25,0.5$, and $1 \mathrm{nM}$. Each concentration was set at least five parallel wells. Then, the cells were cultured constantly for 3 days. CCK- 8 assay was used on $\mathrm{H} 1299$ at $4 \mathrm{~h}, 1,2$, and 3 days according to the manufacturer's instructions. The absorbance was measured spectrophotometrically by using an enzyme-linked immunosorbent assay microplate reader (Synergy 2, Bio-TEK).

High-speed and multiplexed Raman cell imaging. First, the cells were allowed to adhere to the quartz bottomed plates for at least $24 \mathrm{~h}$. For ultrafast cell imaging purpose, cells were then incubated with P-GERTs at a final concentration of $0.05 \mathrm{nM}$ for $6 \mathrm{~h}$. For multiplexed cell imaging, cells were incubated with GERTs with different external Raman reporters (4-MBN, 3-FTP, 3,4-DBT or 2-NT) at a final concentration of $0.05 \mathrm{nM}$ for $6 \mathrm{~h}$. After that cells were washed extensively with phosphate buffered saline (PBS) and fixed with $4 \%$ paraformaldehyde for $10 \mathrm{~min}$ at room temperature. Excess paraformaldehyde was removed by deionized water and the samples were air-dried before SERS measurements. Cell Raman imaging was performed in a step-by-step mapping mode (Xplora INV, Horiba). In this mode, the cell samples do not move during mapping, and laser is scanned point by point across the samples through rotation of the DuoScan mirror, which greatly improves the imaging speed. For ultrafast cell imaging, the image of a whole cell was obtained within $6 \mathrm{~s}$ with a laser power of $370 \mu \mathrm{W}$ and $0.7 \mathrm{~ms}$ acquisition time per pixel for $55 \times 66 \mu \mathrm{m}^{2}$ field of view $(50 \times 50$ pixels, $\times 60$ objective lens, and $785 \mathrm{~nm}$ laser). For multiplexed cell imaging, the image of a whole cell was obtained within $43 \mathrm{~s}$ with a laser power of $370 \mu \mathrm{W}$ and $10 \mathrm{~ms}$ acquisition time per pixel for $24 \times 66 \mu \mathrm{m}^{2}$ field of view $(30 \times 83$ pixels, $\times 60$ objective lens, and $785 \mathrm{~nm}$ laser).

High-speed lymph node in situ Raman imaging. The nude mice (female, 6 weeks) from Shanghai Laboratory Animal Research Center (SLARC, Shanghai, China) were used and cultured under specific pathogen-free (SPF) conditions. All animal experiments were approved by the Animal Care and Use Committee of Shanghai Jiao Tong University. Mice $(n=3)$ were injected subcutaneously with P-GERTs in saline $(25 \mu \mathrm{L}$, $0.5 \mathrm{mg} / \mathrm{mL}$ ) in the paw of the right hind limb. After $24 \mathrm{~h}$, the mice were anesthetized and ultrafast lymph nodes in situ Raman imaging were performed with $785 \mathrm{~nm}$ laser, $\times 10$ objective lens, and $370 \mu \mathrm{W}$ laser power. In order to minimize the time required for large-area sample imaging, we chose Macro mapping mode for lymph node imaging. During Macro mapping mode, the laser beam is raster-scanned to record an average spectrum across a big area, and the sample is moved by the motorized stage with a step matched to the scanned area size to cover the whole surface. In our experiment, the scanning area was $3.2 \times 2.8 \mathrm{~cm}^{2}$, and the motorized stage is moved with a step of $600 \mu \mathrm{m}$ and raster-scanning by laser beam is performed at the same time to ensure that All Raman signals in the area of $600 \times 600 \mu \mathrm{m}^{2}$ are collected. The acquisition time can be shortened to $0.7 \mathrm{~ms}$ per pixel, and the whole Raman image can be obtained within $52 \mathrm{~s}(20 \times 20$ pixels $)$. It is worth noting that, each pixel contains the information over a large area of $600 \times 600 \mu \mathrm{m}^{2}$, which is tens of thousands of times larger than the normal laser spot area. This allows us to collect sample information as much as possible with short imaging time and few pixels. All the Raman reconstruction images were generated with the characteristic band of P-GERTs at $1340 \mathrm{~cm}^{-1}$ by using the LabSpec 6 software.

Reporting summary. Further information on research design is available in the Nature Research Reporting Summary linked to this article.

\section{Data availability}

Data are available within the article and supplementary files. The source data underlying Fig. 2a, d, and Supplementary Figs. $2 \mathrm{c}$ and 4 are provided as a Source Data file. All other data that support the findings of the study are available from the corresponding author upon reasonable request.

Received: 15 January 2019 Accepted: 7 August 2019 Published online: 29 August 2019

\section{References}

1. Sha, M. Y., Xu, H., Natan, M. J. \& Cromer, R. Surface-enhanced raman scattering tags for rapid and homogeneous detection of circulating tumor cells in the presence of human whole blood. J. Am. Chem. Soc. 130, 17214-17215 (2008).

2. Ding, S.-Y., You, E.-M., Tian, Z.-Q. \& Moskovits, M. Electromagnetic theories of surface-enhanced raman spectroscopy. Chem. Soc. Rev. 46, 4042-4076 (2017)

3. Kneipp, J., Kneipp, H., McLaughlin, M., Brown, D. \& Kneipp, K. In vivo molecular probing of cellular compartments with gold nanoparticles and nanoaggregates. Nano Lett. 6, 2225-2231 (2006).

4. Kang, J. W., So, P. T., Dasari, R. R. \& Lim, D. High resolution live cell raman imaging using subcellular organelle-targeting SERS-sensitive gold nanoparticles with highly narrow intra-nanogap. Nano Lett. 15, 1766-1772 (2015)

5. Huefner, A. et al. Characterization and visualization of vesicles in the endolysosomal pathway with surface-enhanced raman spectroscopy and chemometrics. ACS Nano 10, 307-316 (2016)

6. Kircher, M. F. et al. A brain tumor molecular imaging strategy using a new triple-modality mri-photoacoustic-raman nanoparticle. Nat. Med. 18, 829-834 (2012)

7. Spaliviero, M. et al. Detection of lymph node metastases with serrs nanoparticles. Mol. Imaging Biol. 18, 677-685 (2016).

8. Wang, Y., Yan, B. \& Chen, L. SERS tags: Novel optical nanoprobes for bioanalysis. Chem. Rev. 113, 1391-1428 (2013).

9. Pei, H., Zuo, X., Zhu, D., Huang, Q. \& Fan, C. Functional DNA nanostructures for theranostic applications. Acc. Chem. Res. 47, 550-559 (2014).

10. Aberasturi, D. et al. Surface enhanced raman scattering encoded gold nanostars for multiplexed cell discrimination. Chem. Mater. 28, 6779-6790 (2016).

11. Yang, G. et al. Facile synthesis of black phosphorus-au nanocomposites for enhanced photothermal cancer therapy and surface-enhanced raman scattering analysis. Biomater. SCI-UK 5, 2048-2055 (2017).

12. Chen, H. et al. Fabrication of graphene and aunp core polyaniline shell nanocomposites as multifunctional theranostic platforms for SERS real-time monitoring and chemo-photothermal therapy. Theranostics 6, 1096-1104 (2016).

13. Ehlerding, E. B., Chen, F. \& Cai, W. Biodegradable and renal clearable inorganic nanoparticles. Adv. Sci. 3, 1500223 (2016).

14. Harmsen, S. et al. Surface-enhanced resonance raman scattering nanostars for high-precision cancer imaging. Sci., Transl. Med. 7, 271 ra277 (2015)

15. Nayak, T. R. et al. Tissue factor-specific ultra-bright serrs nanostars for raman detection of pulmonary micrometastases. Nanoscale 9, 1110-1119 (2017).

16. Andreou, C. et al. Imaging of liver tumors using surface-enhanced raman scattering nanoparticles. ACS Nano 10, 5015-5026 (2016).

17. Huang, R. et al. High precision imaging of microscopic spread of glioblastoma with a targeted ultrasensitive serrs molecular imaging probe. Theranostics $\mathbf{6}$, 1075-1084 (2016)

18. Fabris, L. SERS tags: The next promising tool for personalized cancer detection? ChemNanoMat 2, 249-258 (2016).

19. Lim, D. K. et al. Highly uniform and reproducible surface-enhanced raman scattering from DNA-tailorable nanoparticles with 1-nm interior gap. Nat. Nanotechnol. 6, 452-460 (2011). 
20. Cassano, D., Pocoví-Martínez, S. \& Voliani, V. Ultrasmall-in-nano approach: Enabling the translation of metal nanomaterials to clinics. Bioconjugate Chem. 29, 4-16 (2018).

21. Cialla-May, D., Zheng, X. S., Weber, K. \& Popp, J. Recent progress in surfaceenhanced raman spectroscopy for biological and biomedical applications: From cells to clinics. Chem. Soc. Rev. 46, 3945-3961 (2017).

22. Qian, X. et al. In vivo tumor targeting and spectroscopic detection with surface-enhanced raman nanoparticle tags. Nat. Biotechnol. 26, 83-90 (2007).

23. Mohs, A. M. et al. Hand-held spectroscopic device for in vivo and intraoperative tumor detection: Contrast enhancement, detection sensitivity, and tissue penetration. Anal. Chem. 82, 9058-9065 (2010).

24. Neuschmelting, V. et al. Dual-modality surface-enhanced resonance raman scattering and multispectral optoacoustic tomography nanoparticle approach for brain tumor delineation. Small 14, 1800740 (2018).

25. Gao, X. et al. Guiding brain-tumor surgery via blood-brain-barrier-permeable gold nanoprobes with acid-triggered mri/serrs signals. Adv. Mater. 29, 1603917 (2017).

26. Zeng, L. et al. Raman reporter-coupled agcore@aushell nanostars for in vivo improved surface enhanced raman scattering imaging and near-infraredtriggered photothermal therapy in breast cancers. ACS Appl. Mater. Interfaces 7, 16781-16791 (2015).

27. Lv, Q. et al. Total aqueous synthesis of au@cu2-xs core-shell nanoparticles for in vitro and in vivo SERS/pa imaging-guided photothermal cancer therapy. Adv. Healthc. Mater. 8, 1801257 (2019).

28. Lin, L., Gu, H. \& Ye, J. Plasmonic multi-shell nanomatryoshka particles as highly tunable SERS tags with built-in reporters. Chem. Commun. 51, 17740-17743 (2015).

29. Lin, L. et al. Nanooptics of plasmonic nanomatryoshkas: Shrinking the size of a core-shell junction to subnanometer. Nano Lett. 15, 6419-6428 (2015).

30. Zhang, Y. et al. Ultraphotostable mesoporous silica-coated gap-enhanced raman tags (gerts) for high-speed bioimaging. ACS Appl. Mater. Interfaces 9, 3995-4005 (2017).

31. Lee, J.-H. et al. Synthesis, optical properties, and multiplexed raman bioimaging of surface roughness-controlled nanobridged nanogap particles. Small 12, 4726-4734 (2016).

32. Ayala-Orozco, C. et al. Au nanomatryoshkas as efficient near-infrared photothermal transducers for cancer treatment: Benchmarking against nanoshells. ACS Nano 8, 6372-6381 (2014).

33. Kumar, A., Kumar, S., Rhim, W.-K., Kim, G.-H. \& Nam, J.-M. Oxidative nanopeeling chemistry-based synthesis and photodynamic and photothermal therapeutic applications of plasmonic core-petal nanostructures. J. Am. Chem. Soc. 136, 16317-16325 (2014).

34. Gandra, N. \& Singamaneni, S. Bilayered raman-intense gold nanostructures with hidden tags (brights) for high-resolution bioimaging. Adv. Mater. 25, 1022-1027 (2013).

35. Kim, M. et al. Dealloyed intra-nanogap particles with highly robust, quantifiable surface-enhanced raman scattering signals for biosensing and bioimaging applications. ACS Cent. Sci. 4, 277-287 (2018).

36. Zhou, J. et al. Polydopamine-enabled approach toward tailored plasmonic nanogapped nanoparticles: from nanogap engineering to multifunctionality. ACS Nano 10, 11066-11075 (2016).

37. Song, J. et al. SERS-encoded nanogapped plasmonic nanoparticles: Growth of metallic nanoshell by templating redox-active polymer brushes. J. Am. Chem. Soc. 136, 6838-6841 (2014).

38. Li, J. et al. Surface-enhanced raman scattering active plasmonic nanoparticles with ultrasmall interior nanogap for multiplex quantitative detection and cancer cell imaging. Anal. Chem. 88, 7828-7836 (2016).

39. Khlebtsov, B. N. \& Khlebtsov, N. G. Surface morphology of a gold core controls the formation of hollow or bridged nanogaps in plasmonic nanomatryoshkas and their SERS responses. J. Phys. Chem. C. 120, 15385-15394 (2016).

40. Bao, Z. et al. Gap-enhanced raman tags for high-contrast sentinel lymph node imaging. Biomaterials 163, 105-115 (2018).

41. Lukianova-Hleb, E. Y. et al. Intraoperative diagnostics and elimination of residual microtumours with plasmonic nanobubbles. Nat. Nanotechnol. 11, 525-532 (2016).

42. Qiu, Y. et al. Intraoperative detection and eradication of residual microtumors with gap-enhanced raman tags. ACS Nano 12, 7974-7985 (2018).

43. Berkels, B., Cabrilo, I., Haller, S., Rumpf, M. \& Schaller, K. Co-registration of intra-operative brain surface photographs and pre-operative $\mathrm{mr}$ images. Int. J. Comput. Assist. Radiol. Surg. 9, 387-400 (2014).

44. Jiang, C., Wang, Y., Song, W. \& Lu, L. Delineating the tumor margin with intraoperative surface-enhanced raman spectroscopy. Anal. Bioanal. Chem. 411, 3993-4006 (2019).

45. Park, J.-E., Kim, M., Hwang, J.-H. \& Nam, J.-M. Golden opportunities: plasmonic gold nanostructures for biomedical applications based on the second near-infrared window. Small. Methods 1, 1600032 (2017).
46. Lin, L., Liu, Z., Li, X., Gu, H. \& Ye, J. Quantifying the reflective index of nanometer-thick thiolated molecular layers on nanoparticles. Nanoscale $\mathbf{9}$, 2213-2218 (2017)

47. Sergiienko, S., Moor, K., Gudun, K., Yelemessova, Z. \& Bukasov, R. Nanoparticle-nanoparticle vs. nanoparticle-substrate hot spot contributions to the SERS signal: Studying raman labelled monomers, dimers and trimers. Phys. Chem. Chem. Phys. 19, 4478-4487 (2017).

48. Lane, L. A., Xue, R. \& Nie, S. Emergence of two near-infrared windows for in vivo and intraoperative SERS. Curr. Opin. Chem. l Biol. 45, 95-103 (2018).

49. Vijayaraghavan, P., Vankayala, R., Chiang, C.-S., Sung, H.-W. \& Hwang, K. C Complete destruction of deep-tissue buried tumors via combination of gene silencing and gold nanoechinus-mediated photodynamic therapy. Biomaterials 62, 13-23 (2015).

50. Chang, H.-H. \& Murphy, C. J. Mini gold nanorods with tunable plasmonic peaks beyond $1000 \mathrm{~nm}$. Chem. Mater. 30, 1427-1435 (2018).

51. Li, J. F. et al. Shell-isolated nanoparticle-enhanced raman spectroscopy. Nature 464, 392 (2010).

52. Zhang, H. et al. In situ dynamic tracking of heterogeneous nanocatalytic processes by shell-isolated nanoparticle-enhanced raman spectroscopy. Nat. Commun. 8, 15447 (2017)

53. Kneipp, K. et al. Single molecule detection using surface-enhanced raman scattering (SERS). Phys. Rev. Lett. 78, 1667 (1997).

54. Maeda, H. The enhanced permeability and retention (EPR) effect in tumor vasculature: The key role of tumor-selective macromolecular drug targeting. Adv. Enzym. Regul. 41, 189-207 (2001).

55. Zhang, Y. et al. Intraoperative raman-guided chemo-photothermal synergistic therapy of advanced disseminated ovarian cancers. Small 14, 1801022 (2018).

56. Kearns, H., Shand, N. C., Smith, W. E., Faulds, K. \& Graham, D. 1064 nm SERS of nir active hollow gold nanotags. Phys. Chem. Chem. Phys. 17, 1980-1986 (2015)

57. Kearns, H. et al. Sensitive SERS nanotags for use with a hand-held $1064 \mathrm{~nm}$ raman spectrometer. Roy. Soc. Open Sci. 4, 170422 (2017).

58. Song, J., Pu, L., Zhou, J., Duan, B. \& Duan, H. Biodegradable theranostic plasmonic vesicles of amphiphilic gold nanorods. ACS Nano 7, 9947-9960 (2013).

59. Song, J. et al. Ultrasmall gold nanorod vesicles with enhanced tumor accumulation and fast excretion from the body for cancer therapy. $A d v$. Mater. 27, 4910-4917 (2015).

60. Gorelikov, I. \& Matsuura, N. Single-step coating of mesoporous silica on cetyltrimethyl ammonium bromide-capped nanoparticles. Nano Lett. 8, 369-373 (2008).

61. Johnson, P. B. \& Christy, R. W. Optical constants of the noble metals. Phys. Rev. B 6, 4370-4379 (1972).

62. Lumerical. Overcoming the Multi-wavelength FDTD Challenge. https://www. lumerical.com/learn/whitepapers/overcoming-the-multi-wavelength-fdtdchallenge/ (2019).

63. Zhuang, X., Miranda, P. B., Kim, D. \& Shen, Y. R. Mapping molecular orientation and conformation at interfaces by surface nonlinear optics. Phys. Rev. B 59, 12632-12640 (1999).

64. Cecchet, F. et al. Orientational analysis of dodecanethiol and p-nitrothiophenol sams on metals with polarisation-dependent sfg spectroscopy. ChemPhysChem 11, 607-615 (2010).

65. Le Ru, E. C., Blackie, E., Meyer, M. \& Etchegoin, P. G. Surface enhanced raman scattering enhancement factors: a comprehensive study. J. Phys. Chem. C. 111, 13794-13803 (2007)

66. Pugmire, D. L., Tarlov, M. J., van Zee, R. D. \& Naciri, J. Structure of 1,4benzenedimethanethiol self-sssembled monolayers on gold grown by solution and vapor techniques. Langmuir 19, 3720-3726 (2003).

\section{Acknowledgements}

We acknowledge the financial support from National Natural Science Foundation of China (Nos. 81571763, 81622026, 61425006, and 81871401), Shanghai Jiao Tong University (Nos. YG2016MS51 and YG2017MS54), the State Key Laboratory of Oncogenes and Related Genes (No. 91-17-28), Shanghai Key Laboratory of Gynecologic Oncology, Guangci Professorship Program of Ruijin Hospital, and Innovation Research Plan supported by Shanghai Municipal Education Commission (No. ZXWF082101).

\section{Author contributions}

Y.Q.Z. and J.Y. conceived the idea for this work and designed the experiments. Y.Q.Z and Y.Q.G. performed the experiments. J.H. contributed to the numerical calculations. Y.Q.Z., Y.Q.G., B.D.T. and J.Y. wrote and revised the paper. All authors discussed the results and commented on the paper. 


\section{Additional information}

Supplementary Information accompanies this paper at https://doi.org/10.1038/s41467019-11829-y.

Competing interests: The authors declare no competing interests.

Reprints and permission information is available online at http://npg.nature.com/ reprintsandpermissions/

Peer review information: Nature Communications thanks Dorleta Jimenez de Aberasturi and other anonymous reviewer(s) for their contribution to the peer review of this work. Peer reviewer reports are available.

Publisher's note: Springer Nature remains neutral with regard to jurisdictional claims in published maps and institutional affiliations. (c) (i) Open Access This article is licensed under a Creative Commons Attribution 4.0 International License, which permits use, sharing, adaptation, distribution and reproduction in any medium or format, as long as you give appropriate credit to the original author(s) and the source, provide a link to the Creative Commons license, and indicate if changes were made. The images or other third party material in this article are included in the article's Creative Commons license, unless indicated otherwise in a credit line to the material. If material is not included in the article's Creative Commons license and your intended use is not permitted by statutory regulation or exceeds the permitted use, you will need to obtain permission directly from the copyright holder. To view a copy of this license, visit http://creativecommons.org/ licenses/by/4.0/

(C) The Author(s) 2019 